\title{
Attitudes toward Mental Illness among Minority Freshmen in China
}

\author{
Wei-yu Zeng ${ }^{1}$, Xiao-xue Xie ${ }^{1}$, Xiang-rong Tang ${ }^{1}$, Meng-long Wang ${ }^{1}$, \\ Lan Yang ${ }^{1}$, Ying-ying Ning ${ }^{1} \&$ Xiao-gang Wang ${ }^{1}$ \\ ${ }^{1}$ School of Sociology and Psychology, Southwest Minzu University, Chengdu, China \\ Correspondence: Xiao-gang Wang, School of Sociology and Psychology, Southwest Minzu University, Chengdu, \\ China. E-mail: xiaogangwang2011@163.com
}

Received: November 3, 2019

doi:10.5539/ijps.v11n4p96
Accepted: November 18, 2019 Online Published: November 19, 2019

URL: https://doi.org/10.5539/ijps.v11n4p96

\begin{abstract}
To explore the status and influencing factors of attitudes toward mental illness among minority freshmen and provide references for mental health education and service assistance in ethnic colleges, a questionnaire survey was conducted among 581 minority freshmen by using Mental Illness Emotional Scale, Stereotype Scale and Social Distance Scale from autumn of 2018 to next spring. The scores of minority freshmen on these scales were significantly higher than the theoretical median, which demonstrated that they did have negative attitudes. There were the significant main effect of nation on the anger and sympathy dimensions of Mental Illness Emotional Scale while the same results were found in the main effect of gender on the danger and suicidal behavior dimensions of Mental Illness Stereotype Scale and the main effect of nation on the dimension of dependence consciousness. Whether relatives or friends are mental illness patients had significant effect on the suicidal behavior dimension of Mental Illness Stereotypes Scale. Overall, attitudes toward mental illness were negative and some mental illness stigma phenomenon were found among minority freshmen; ethnic was an important factor affecting the attitudes of minority freshmen toward mental illness, and the inter-group contact with patients who suffer mental illness in life had a slight impact on the attitudes toward mental illness.
\end{abstract}

Keywords: mental illness, attitude, freshman, nation

\section{Introduction}

Attitude toward mental illness refers to the attitudes of individuals to mental illness and mental illness patients and can be divided into positive and negative attitude. Negative attitude are the so-called mental illness stigma (Du, Xu, Wang \& Lan, 2017; Wang, Jackson, Huang \& Chen, 2012). Mental illness stigma or negative attitudes are important obstacles in people's mental health maintenance process (Corrigan, 2004; 2005). Reducing or decreasing the impact of mental illness stigma or negative attitude have always been an important issue in the field of mental health service.

Some studies have found that the demand of college students for professional psychological aid institutions was low, such as psychological counseling centers in colleges. It has also been discovered that only $1.46 \%$ of students were willing to seek professional psychological assistance (Feng, 2018). In Chinese colleges, freshmen have always been the starting point and the key part of school mental health education and service. Stepping into the new campus, they often face various adaptation problems, and have higher incidence of psychological problems comparing with other college students. Additionally, it is a critical period for freshmen when their psychological developments are maturing at the same time (Yin, 2017). It is worrisome that studies have shown that the psychological help-seeking attitude of freshmen was significantly lower than other grades (Guo, Zhu, 2019; Li, 2016). There are multiple cultural differences in ethnic colleges which are called comprehensive ordinary colleges established by the party and the state to solve Chinese domestic ethnic problems, such as different regions, religious beliefs, and customs. Minority freshmen who do not belong to main ethnic group therefore are more likely to face adaptation problems, and the incidence of psychological problems may be higher (Yu, 2015). How to help minority students overcome adaptation disorders caused by various factors and strengthen the improvement of mental health education has become a key research topic in ethnic colleges (Liang, Shi, Zhang, Wang \& Basang, 2018).

In order to improve mental health education and services in ethnic colleges, most of the researches focus on mental health needs, while ignore the importance and harmfulness of mental illness stigma. At the same time, 
previous studies have less explored the attitudes toward mental illness of minority students, which is not conducive for schools and teachers to carrying out targeted mental health education works. Therefore, this study aims to explore the status and influencing factors of attitudes toward mental illness of minority freshmen, and provide references for mental health education and services in ethnic colleges.

\section{Objects and Methods}

\subsection{Objects}

A survey on attitude toward mental health with 667 participants among the freshmen in an ethnic college called Southwest Minzu University was conducted from autumn of 2018 to next spring. The effective questionnaires were 581 , and the effective recovery rate was $87 \%$.

\subsection{Tools}

Classic Attitude ABC model theory proposed that attitudes consist of cognitive, emotional, and behavioral tendency of individuals toward attitude objects (Dovidio, Kawakami, \& Gaertner, 2002). According to the model and drawing on the previous researches on attitude toward mental illness, this study used revised Mental Illness Emotion Scale, Stereotype Scale, Social Distance Scale by Zhou (2006) to examine emotion, recognition, and behavior, which were three components of attitude with mental illness.

\subsubsection{Emotion Scale}

Emotion Scale was revised by Zhou who selected the topics to measure emotion from the Attribution Questionnaire designed by Corrigan et al., which is to measure the emotional response of subjects to mental illness patients. The scale contains three dimensions of anger, sympathy and fear. The higher the score of the subject is, the more likely they are to feel angry, afraid or sympathetic to the mentally ill person, and the more negative they are toward the mentally ill person. The reliability of the scale in this survey was 0.75 and the validity was 0.79 .

\subsubsection{Stereotype Scale}

Stereotype Scale contains three scales of dependency awareness, danger and suicidal behavior. The Chinese version was translated and revised by Zhou (2006). Among them, Dependency Awareness Scale includes 3 topics, and the higher the score is, the more the subject considers the patient to be dependent. Danger Scale also includes 3 topics. The higher the score is, the more the subject believes that the patient may be dangerous to others. And Suicidal Behavior Scale has a total of 4 topics. The higher the total scores are, the more the subject believes that the patient is at risk for suicide. The scale had a reliability of 0.80 and a validity of 0.82 in this study.

\subsubsection{Social Distance Scale}

Social Distance Scale was developed by Bogardue. It was used to measure the social distance caused by ethnicity. It was widely used to change the public's attitude towards mental illness and to measure the degree of discrimination that might be caused by patients with mental illness. The scale has 7 questions. The higher the score of the questionnaire is, the more the participant wants to maintain a social distance from the patient, that is, the higher the tendency to discrimination (Link, Yang, \& Phelan, 2004). The reliability of the scale in this survey was 0.85 and the validity was 0.79 .

\subsection{Data Collection and Processing}

The survey was class-based and conducted collectively, and all questionnaires were distributed and collected on the spot. The data was recorded, purified and analyzed using SPSS 23.0 statistical software. While performing statistical descriptions, the single-sample t-test, independent sample t-test, and multi-factor analysis of variance were used to test whether the average score of each dimension of the scale was statistically significant.

\section{Results}

\subsection{Basic Situation of Freshmen}

Descriptive statistical analysis showed that among 581 freshmen, 234 were aged 18 and under, 231 were aged 19, and 116 were 20 and over. In terms of gender distribution, there are 179 males and 402 females. In the ethnic distribution, 209 were tested by the Yi people, 172 were tested by the Tibetans, and 200 were the Hui people (see Table 1). 
Table 1. Demographic characteristics of freshmen

\begin{tabular}{ccccccccc}
\hline & \multicolumn{3}{c}{ Age } & \multicolumn{2}{c}{ Gender } & \multicolumn{3}{c}{ Nation } \\
\cline { 2 - 9 } & $\leq 18$ & $=19$ & $\geq 20$ & Male & Female & Yi $^{l}$ & Tibetan $^{2}$ & Hui $^{3}$ \\
\hline Number & 234 & 231 & 116 & 179 & 402 & 209 & 172 & 200 \\
Proportion & 0.40 & 0.40 & 0.20 & 0.31 & 0.69 & 0.36 & 0.30 & 0.34 \\
\hline
\end{tabular}

Note. 1=the $Y i$ nationalities, one of the ethnic groups in China, mainly distributed over Yunnan, Sichuan and Guizhou; 2=one of the oldest ethnic groups in China and South Asia and Tibetans are indigenous peoples of the Qinghai-Tibet Plateau and believe in Tibetan Buddhism mainly distributing in the Tibet Autonomous Region, Qinghai Province and the western part of Sichuan Province; 3=the Hui nationalities, one of the ethnic groups in China, mainly distributed over Ningxia, Gansu, and Henan.

\subsection{Analysis of the Overall Level of Attitudes towards Mental Illness in Freshmen}

Single-sample t-test showed that the average scores of the freshmen on the anger and fear dimensions in Mental Illness Emotion Scale were significantly lower than the theoretical median $3(p<.05)$, as well as the danger dimension in Stereotype Scale $(p<.05)$. The average scores of sympathy dimension in Emotion Scale, dependency awareness dimension in Stereotype Scale, and Social Distance Scale were significantly higher than the theoretical median $3(p<.05)$, while there was no significant difference $(p>.05)$ between the average score of suicidal behavior dimension and the theoretical median 3 (see Table 2).

Table 2. The overall difference of attitudes toward mental illness among freshmen

\begin{tabular}{|c|c|c|c|c|c|c|c|}
\hline & \multicolumn{3}{|c|}{ Emotion Scale } & \multicolumn{3}{|c|}{ Stereotype Scale } & \multirow{2}{*}{$\begin{array}{c}\text { Social } \\
\text { Distance } \\
\text { Scale }\end{array}$} \\
\hline & Anger & Sympathy & Fear & $\begin{array}{c}\text { Dependency } \\
\text { awareness }\end{array}$ & Danger & $\begin{array}{l}\text { Suicidal } \\
\text { behavior }\end{array}$ & \\
\hline$M$ & 2.17 & 3.37 & 2.38 & 3.27 & 2.83 & 3.02 & 3.24 \\
\hline$S D$ & 0.91 & 0.90 & 0.92 & 0.75 & 0.89 & 0.80 & 0.77 \\
\hline$t$ & $-21.81 * * *$ & $9.99 * * *$ & $-16.22 * * *$ & $8.72 * * *$ & $-4.62 * * *$ & 0.65 & $7.64^{* * *}$ \\
\hline
\end{tabular}

Note. ${ }^{* * *}=p<.001$.

\subsection{Analysis of Freshmen's Attitudes toward Mental Illness of Different Types}

Statistical analysis found that in Mental Illness Emotion Scale, the main effects of nation on the anger and sympathy dimensions were significant $\left(F_{(1,5)}=13.44, p<.001 ; F_{(1,5)}=4.89, p<.01\right)$. In Mental Illness Stereotype Scale, the main effect of gender was significant on the danger and suicidal behavior dimensions $\left(F_{(1,5)}\right.$ $\left.=4.95, p<.05 ; F_{(1,5)}=4.52, p<.05\right)$, the same as the main effect of nation on the dependency awareness $\left(F_{(1,5)}=\right.$ $3.98, p<.01)$. But it showed that the main effects of gender and nation had no significance on the social distance in Social Distance Scale.

Table 3. Mean of different types of attitudes toward mental illness among freshmen

\begin{tabular}{|c|c|c|c|c|c|c|c|c|c|c|c|c|c|c|}
\hline & & \multicolumn{5}{|c|}{ Emotion Scale } & \multicolumn{6}{|c|}{ Stereotype Scale } & \multirow{2}{*}{\multicolumn{2}{|c|}{$\begin{array}{c}\text { Social } \\
\text { Distance } \\
\text { Scale }\end{array}$}} \\
\hline & & \multicolumn{2}{|c|}{ Fear } & \multicolumn{2}{|c|}{ Anger } & Sympathy & \multicolumn{2}{|c|}{$\begin{array}{c}\text { Dependency } \\
\text { awareness }\end{array}$} & \multicolumn{2}{|c|}{ Danger } & \multicolumn{2}{|c|}{$\begin{array}{l}\text { Suicidal } \\
\text { behavior }\end{array}$} & & \\
\hline & & $M$ & $S D$ & $M$ & $S D$ & $M \quad S D$ & $M$ & $S D$ & $M$ & $S D$ & $M$ & $S D$ & $M$ & $S D$ \\
\hline \multirow{3}{*}{ Gender } & Male & 2.40 & 0.93 & 2.20 & 0.87 & 3.350 .91 & 3.20 & 0.74 & 2.70 & 0.91 & 2.93 & 0.80 & 3.20 & 0.80 \\
\hline & Female & 2.38 & 0.91 & 2.17 & 0.93 & 3.390 .90 & 3.30 & 0.76 & 2.89 & 0.88 & 3.06 & 50.79 & 3.26 & 0.75 \\
\hline & $Y i$ & 2.42 & 0.86 & 2.39 & 0.97 & 3.181 .00 & 3.15 & 0.80 & 2.89 & 0.86 & 2.93 & 0.81 & 3.30 & 0.76 \\
\hline \multirow[t]{2}{*}{ Nation } & Tibetan & 2.47 & 0.90 & 2.22 & 0.85 & 3.560 .80 & 3.41 & 0.73 & 2.86 & 0.87 & 3.07 & 0.80 & 3.21 & 0.72 \\
\hline & Hui & 2.27 & 0.97 & 1.91 & 0.83 & 3.420 .85 & 3.28 & 0.70 & 2.74 & 0.94 & 3.07 & 0.78 & 3.22 & 0.82 \\
\hline
\end{tabular}


Multiple comparisons showed that the average score of males was significantly lower than that of females on the dimensions of danger and suicidal behavior in Mental Illness Stereotypes Scale $(p<.05)$. On other scales, there was no significant difference in the scores of males and females. In the anger dimension in Mental Illness Emotion Scale, the average score of $Y i$ freshmen was the highest, and the average scores of $Y i$ and Tibetan freshmen were significantly higher than those of Hui freshmen $(p<.01)$. In the sympathy dimension, the average score of Tibetan was the highest, while the scores of Hui and Tibetan freshmen were significantly higher than those of $Y i$ freshmen $(p<.01)$. And on the dependency awareness dimension in Mental Illness Stereotypes Scale, Tibetan freshmen had the highest average score, which was higher than the average score of $Y i$ freshmen ( $p$ $<.01)$. In Mental Illness Social Distance Scale, there were no significant difference among the average scores of $Y i$, Tibetan and Hui Freshmen.

\subsection{Analysis of Attitudes toward Mental Illness of Freshmen in Different Inter-group Contact}

Studies have found that intergroup contact is an important factor to affect attitude toward mental illness (Wang, 2013). This study tried to learn about the contacts of the subjects with mental illness patients in their life from 3 aspects which were whether to receive mental counseling or treatment, whether relatives or friends are mental illness patients and whether friends and classmates have mental illness. But analysis of variance only showed found that whether relatives or friends are mental illness patients had significant effect on the suicidal behavior dimension of Mental Illness Stereotypes $\left(F_{(1,7)}=3.90, p<.05\right)$, and there was no significant effect of other inter-group contacts on the remaining scales.

Table 4. Analysis of attitudes toward mental illness among freshmen of different inter-group contact

\begin{tabular}{|c|c|c|c|c|c|c|c|c|c|c|c|c|c|c|c|}
\hline & & \multicolumn{6}{|c|}{ Emotion Scale } & \multicolumn{6}{|c|}{ Stereotype Scale } & \multirow{2}{*}{\multicolumn{2}{|c|}{$\begin{array}{c}\text { Social } \\
\text { Distance } \\
\text { Scale }\end{array}$}} \\
\hline & & \multicolumn{2}{|c|}{ Fear } & \multicolumn{2}{|c|}{ Anger } & \multicolumn{2}{|c|}{ Sympathy } & \multicolumn{3}{|c|}{$\begin{array}{c}\text { Dependency } \\
\text { awareness }\end{array}$} & Danger & \multicolumn{2}{|c|}{$\begin{array}{l}\text { Suicidal } \\
\text { behavior }\end{array}$} & & \\
\hline & & $M$ & $S D$ & $M$ & $S D$ & $M$ & $S D$ & $M$ & $S D$ & $M$ & $S D$ & $M$ & $S D$ & $M$ & $S D$ \\
\hline \multirow{2}{*}{$\begin{array}{l}\text { Whether to } \\
\text { receive mental } \\
\text { counseling or } \\
\text { treatment }\end{array}$} & Yes & 2.33 & 0.99 & 2.23 & 0.93 & 3.12 & 0.80 & 3.17 & 0.65 & 2.79 & 90.83 & 3.09 & 0.93 & 2.98 & 0.86 \\
\hline & No & 2.39 & 0.91 & 2.17 & 0.91 & 3.40 & 0.91 & 3.28 & 0.76 & 2.83 & 30.90 & 3.02 & 0.79 & 3.27 & 0.76 \\
\hline $\begin{array}{l}\text { Relatives or } \\
\text { friends are }\end{array}$ & Yes & 2.29 & 1.12 & 2.06 & 1.03 & 3.32 & 0.79 & 3.24 & 0.86 & 2.50 & 1.14 & 3.23 & 0.89 & 3.09 & 0.92 \\
\hline $\begin{array}{c}\text { mental illness } \\
\text { patients }\end{array}$ & No & 2.39 & 0.91 & 2.18 & 0.91 & 3.38 & 0.91 & 3.27 & 0.75 & 2.84 & 40.88 & 3.01 & 0.79 & 3.25 & 0.76 \\
\hline $\begin{array}{l}\text { Friends and } \\
\text { classmates }\end{array}$ & Yes & 2.38 & 1.02 & 2.08 & 0.92 & 3.38 & 0.88 & 3.28 & 0.80 & 2.74 & 41.03 & 3.24 & 0.90 & 3.12 & 0.93 \\
\hline $\begin{array}{l}\text { have mental } \\
\text { illness }\end{array}$ & No & 2.38 & 0.90 & 2.19 & 0.91 & 3.37 & 0.91 & 3.27 & 0.74 & 2.85 & 50.86 & 2.98 & 0.77 & 3.27 & 0.73 \\
\hline
\end{tabular}

\section{Discussion}

In this study, the attitudes to patients with mental illness are negative among minority freshmen, indicating they have a certain degree of mental illness stigma. Specifically, freshmen feel sympathy for patients with mental illness, without feeling strong anger or fear; they consider that people with mental illness do not pose danger. They also believe that people with mental illness are vulnerable, needing protection and care from others, while they believe this group do not have suicidal tendencies or suicidal behaviors. Although they believe that patients with mental illness need care, they would still keep a certain distance from them, and even show alienation or discrimination in daily interpersonal contact with them. This is also consistent with the results in the survey of attitudes toward mental illness among freshmen in minority colleges (Du et al., 2017).

Generally speaking, both ethnicity and culture are important factors affecting the attitudes toward mental illness of minority freshmen. In this study, $Y i$ freshmen are the most likely to be angry with mental illness patients, followed by the Tibetan and Hui freshmen. Many ethnic minorities in China have various cultural forms such as ancestral beliefs, witch doctors and witchcraft. They often regard some individuals with abnormal mental behaviors as spiritual possession or suppose them possess supernatural powers. These unique culture will affect the attitudes toward mental illness of minority freshmen to varying degrees (Lu, 2012; Tang, 2014). Therefore, the reason why $Y i$ freshmen are the most likely to be angry with mental illness patients probably is that the $Y i$ 
culture does not agree with the notion "mental illness". At the same time, Tibetan freshmen are more likely to feel sympathy for patients. This may be because Tibetans are gradually accustomed to judging everything with the good and evil thought of Buddhist under the influence of Tibetan Buddhism, which forms a social atmosphere of compassion and tolerance (Zhang, 2016). Moreover, Tibetan freshmen are also the easiest to think that the patients are vulnerable and need the care of others, which may also be related to the culture of Tibetan Buddhism requiring monks to keep compassionate bosoms to free suffering beings (Yang, 2011).

What's more, gender is a significant factor in the perception of danger and suicidal behavior. Among minority freshmen, females are more likely than males to consider that patients with mental illness are dangerous and have suicidal behavior. The appearance of such consequence may be related to individual differences between males and females, unique national culture, and current social environment. For example, the idea of "male and female humble" in Tibetan culture is likely to lead girls to have the awareness of a stronger prevention and a higher level of danger to the outside world (Yang, 2011). Otherwise, girls may be more emotional, thoughtful, and sensitive, while men have higher risk-taking qualities (Kou, Feng, Yang, 2018). Therefore, girls are more likely to be at risk of danger. Relatively, more girls believe that suicidal behavior occurred in patients with mental illness, which may be related to the suicidal ideation of girls themselves (Kou et al., 2018).

In addition, the inter-group contact between freshmen and mentally ill patients has less impact on attitudes toward mental illness. The subjects who have received mental counseling or treatment, whose relatives or friends are mental illness patients, and friends and classmates have mental illness will not affect the attitudes to mental illness. However, the subjects whose relatives or friends are mental illness patients are more likely to consider themselves with a tendency to suicide or have suicide behavior. Previous studies have shown that inter-group contact could improve people's attitudes toward mental illness and patients, but not all contacts were effective. Meanwhile, contact situations and the ways of contact also regulated the actual effects of exposure (Corrigan, 2005). In college lives, minority freshmen are unable to enjoy many opportunities to have face-to-face exchanges and equal discussions with relatives, friends, and classmates who have mental illnesses in their lives. So it has difficulty in changing their attitudes toward mental health with patients through simple daily lives.

The study preliminarily reveals the status of attitudes toward mental illness among minority freshmen in China and analyzed them from three aspects of gender, nation and inter-group contact, which may provide new direction for mental health services in ethnic colleges. But one thing to note is that studies have shown that explicit measurement of mental illness stigma based on self-reported scale might not accurately reflect individuals' true attitudes towards mental illness patients (Rüsch, Todd, Bodenhausen, \& Corrigan, 2010; Wang et al., 2012). Due to the number of sample size, the study did not explore the implicit attitude of the subject and the accuracy of the survey results may still have room for improvement. Therefore, researches can consider using the implicit research paradigm to further explore the attitudes toward mental illness of minority freshmen in the future.

\section{References}

Corrigan, P. W. (2004). How stigma interferes with mental healthcare, American Psychologist, 59, 614-625. https://doi.org/10.1037/0003-066X.59.7.614

Corrigan, P. W. (2005). Changing stigma through contact, Advances in Schizophrenia and Clinical Psychiatry, 1, 54-58.

Link, B. G., Yang, L. H. \& Phelan, J. C. (2004). Measuring mental illness stigma, Schizophrenia Bulletin, 30, 511-541. https://doi.org/10.1093/oxfordjournals.schbul.a007098

Rüsch, N., Todd, A., Bodenhausen, G. \& Corrigan, P. W. (2010). Biogenetic models of psychopathology, implicit guilt, and mental illness stigma, Psychiatry Research, 179, 328-332. https://doi.org/10.1016/j.psych res.2009.09.010

Du, L., Xu, C., Wang, X. \& Lan, W. (2017). Investigation on mental illness attitudes among college freshmen, Journal of Guizhou Educational Institute, 33(09), 52-55.

Dovidio, J.F., Kawakami K. \& Gaertner S.L. (2002). Implicit and explicit prejudice and interracial interaction, Journal of Personality and Social Psychology, 82, 62-68. https://doi.org/10.1037/0022-3514.82.1.62

Feng, N. (2018). An Overview of College Students' Mental Illness Stigma, Journal of Tianjin Vocational Institutes, 20(10), 124-128.

Guo, T. \& Zhu, Y. (2019). Study on the Stigma of College Students' Psychological Help, China Journal of Multimedia \& Network Teaching, (03), 122-123. 
Kou, M., Feng, Z. \& Yang, X. (2018). Influencing factors of suicidal ideation of college students: a Meta-analysis, Chinese Preventive Medicine, 19(07), 520-526.

Lu, J. (2012). An Analysis of the Influence of the Yi People's Ancestors' Belief on the Psychological Development of the Yi Nationality, Journal of Southwest University for Nationalities (Humanities and Social Science), 33(03), 47-50.

Liang, J., Shi, H., Zhang, Y., Wang, D. \& Basang, Z. (2018). Research on the Mental Health Status and Education Methods of Minority Freshmen: A Case Study of Minority Students in a University in Xi'an, Medical Education Research and Practice, 26(06), 1006-1009.

Li, Q. (2016). The Study on the Current Situation of College Freshmen Seeking Professional Psychological Help and Education Countermeasures. MA Dissertation. Liaoning University.

Tang, Q. (2014). Religious Folklore and Survival Practice-The Ethnography of Liangshan Yi Adu Village, Religious Culture Press, Beijing.

Wang, X., Yin, T. \& Huang, X. (2012). A Review on Implicit Stigma of Mental Illness, Advances in Psychological Science, 20(3), 384-393. https://doi.org/10.3724/SP.J.1042.2012.00384

Wang, X., Huang, X., Jackson, T. \& Chen, R. (2012). Components of implicit stigma against mental illness among Chinese students, PLOS ONE, 7, 0046016.https://doi.org/10.1371/journal.pone.0046016

Yin, Z. (2017). Research on Current Situation Survey and Influential Factors for the Mental Health of College Students in Nationality Institutes__ A Case Study on South-central University for Nationalities, Bulletin of Sport Science \& Technology, 25(03), 48-49.

Yu, J. (2015). Journal of Sichuan University for Nationalities, Journal of Sichuan University for Nationalities, 24(01), 86-90.

Yang, W. (2011). Influence of Tibetan Buddhism Belief on Tibetan Social Psychology and Behavior, Journal of Southwest University for Nationalities (Humanities and Social Science), 32(02), 17-23.

Zhou, Z. (2006). The Stigma of Depressive Disorder: The Influences of Controllability, Perceived Dangerousness and Perceived Dependency on Public Discrimination, MA Dissertation. Chun Yuan Christian University.

\section{Copyrights}

Copyright for this article is retained by the author(s), with first publication rights granted to the journal.

This is an open-access article distributed under the terms and conditions of the Creative Commons Attribution license (http://creativecommons.org/licenses/by/4.0/). 